\title{
A Case Report of Paraganglioma: A Diagnosis Not to Be Missed
}

\author{
Mohammad Mozaffar ${ }^{1}$, Parima Safe ${ }^{2}$, Tayebeh Rostami ${ }^{3}$, Barmak Gholizadeh (i) ${ }^{4}$ and Adel Zeinalpour \\ (iD) 4 , \\ ${ }^{1}$ Shohadaye Tajrish Hospital, Shahid Beheshti University of Medical Sciences, Tehran, Iran \\ ${ }^{2}$ Clinical Research Center, Loghman Hakim Hospital, Shahid Beheshti University of Medical Sciences, Tehran, Iran \\ ${ }^{3}$ Resident of Pathology, Shohadaye Tajrish Hospital, Shahid Beheshti University of Medical Sciences, Tehran, Iran \\ ${ }^{4}$ Shahid Modarres Hospital, Shahid Beheshti University of Medical Sciences, Tehran, Iran \\ "Corresponding author: Shahid Modarres Hospital, Shahid Beheshti University of Medical Sciences, Velenjak St, Tehran, Iran. Email: adel.zeinalpour@gmail.com
}

Received 2019 November 30; Revised 2020 February 06; Accepted 2020 February 10.

\begin{abstract}
Introduction: The autonomic nervous system, a derivative of neural crest cells, may undergo the neoplastic transformation and give rise to paragangliomas, which can arise either at intra-adrenal or extra-adrenal sites, commonly referred to as pheochromocytomas and paragangliomas, respectively. Although indistinguishable at the cellular level and usually identical in clinical manifestation, these two tumors differ in means of malignancy risk, genetic testing, and the probability of detecting an associated neoplasm, highlighting the importance of making a correct diagnosis to prevent additional complications.

Case Presentation: We report a 17-year-old female patient presented with sudden blurred vision and palpitation lasting for 1 week. The physical examination revealed tachycardia, hypertension, and a mildly tender mass on the left side of the umbilicus. A spiral abdominal and pelvic computed tomography (CT) scan was performed, which depicted a hypervascular retroperitoneal mass in the left infrarenal region. Mildly-elevated urine metanephrine and normetanephrine levels were suggestive of a paraganglioma (PGL). Iodine-131 metaiodobenzylguanidine (MIBG) scan was also in favor of PGL of the organ of Zuckerkandl. The mass was completely resected and the immunohistochemistry (IHC) study of the specimen confirmed the diagnosis.

Conclusions: The diversity of manifestations and rarity of PGL can delay the diagnosis, which may lead to potential complications. This makes it compelling to include PGLs in differential diagnoses in a clinical setting compatible with catecholamine hypersecretion.
\end{abstract}

Keywords: Autonomic Nervous System, Neural Crest, Pheochromocytoma, Paraganglioma of Organ of Zuckerkandl

\section{Introduction}

Paragangliomas (PGLs) are vascular tumors associated with blood vessels and neural structures (1). About $80 \%$ of these tumors occur in the adrenal medulla often termed pheochromocytoma (PCC) (2) and the rest occur at extraadrenal locations. Depending upon location, functional status of the tumor and some other factors, the clinical manifestation of PGLs may differ with some patients even being asymptomatic. Given vague clinical presentation, reaching the diagnosis of a PGL can sometimes be challenging and, thus, its early diagnosis requires a high index of suspicion.

\section{Case Presentation}

A 17-year-old female patient was referred to our emergency department with a sudden blurred vision along with palpitation for 1 week. She had a 2-month history of nausea and a vague, diffuse, and non-radiating abdominal pain. The patient had also experienced occasional restlessness, flushing, and headache for about 1 year. She did not notice any alteration in defecation habits. The medical history, as well as family history, was clear and the patient denied any drug or substance abuse. On the physical examination, she was found to be thin, tachycardic at 110 bpm, and hypertensive with a blood pressure of 190/110 mmHg. Abdominal examination was insignificant other than a mass detected on the left side of the umbilicus with mild tenderness on deep palpation. The electrocardiogram showed tachycardia with normal sinus rhythm and no abnormal changes. An emergent ophthalmology consultation revealed dilated conjunctival vessels and hypertensive retinopathy. The patient underwent spiral abdominal and pelvic computed tomography (CT) scan, which showed a highly hypervascular retroperitoneal mass in the left infrarenal region with a heterogeneous enhancement pattern, tightly fixed to the aorta, surrounding the inlet 
of the aortic bifurcation, and the left iliac artery measuring $12 \times 6 \times 6 \mathrm{~cm}$ (Figure 1). Based on the information provided, the main differential diagnoses consisted of malignant vascular tumors, hypervascular metastases, and extra-adrenal PCCs.
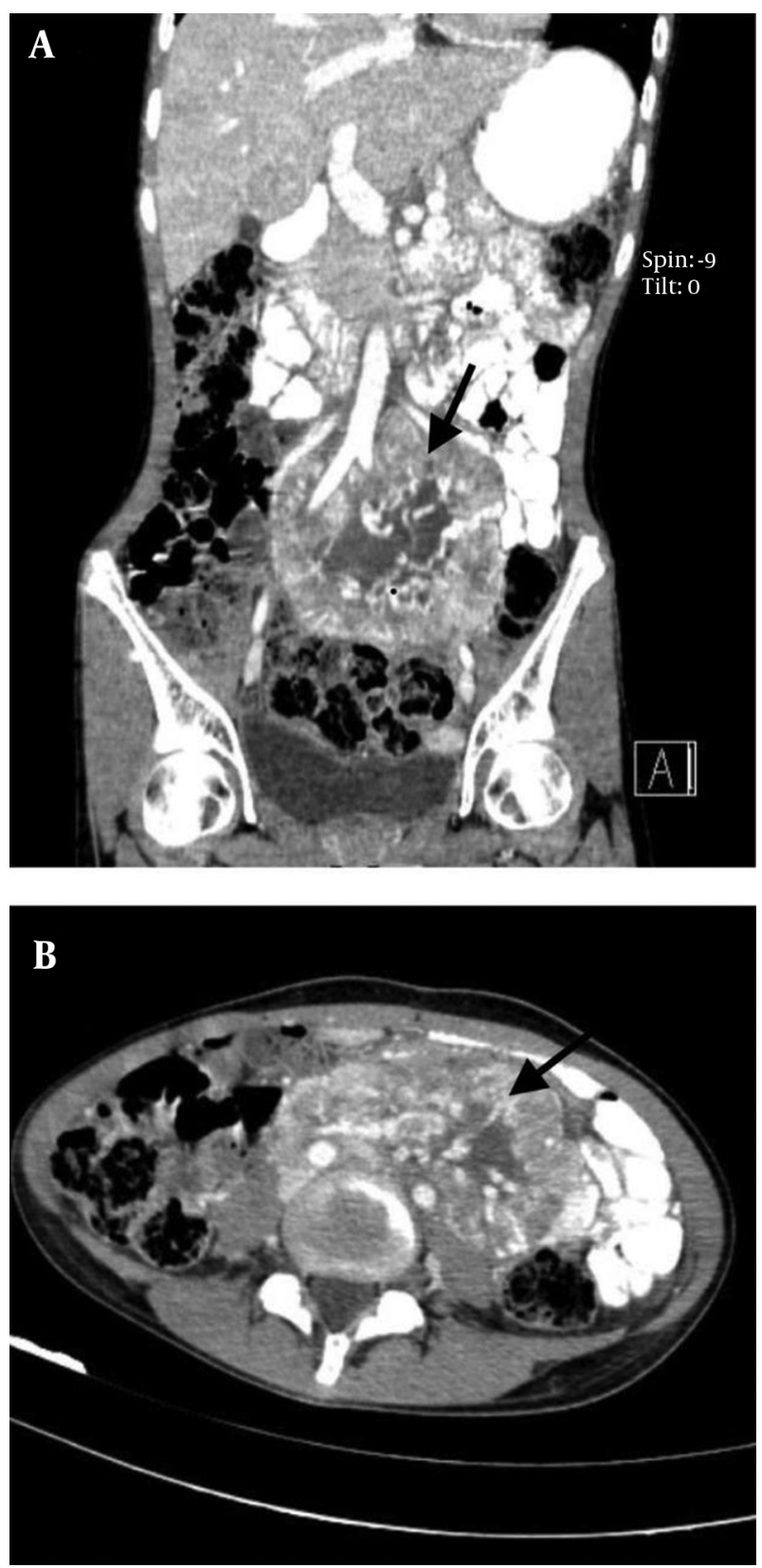

Figure 1. A, Coronal; and B, axial section of the CT scan showing a mass (arrows) with a heterogeneous enhancement pattern surrounding the inlet of the aortic bifurcation and the left iliac artery.

Laboratory testing showed a normal vanillylmandelic acid (VMA) level, mildly-elevated urine metanephrine (1.3, normal range: < 1.3), and urine normetanephrine (82, normal range: 15 - 80) suggestive of a PGL. So, I-131 metaiodobenzylguanidine (MIBG) scan was done, which depicted a curvilinear-shaped area in the abdomen and a large area of increased radiotracer uptake in the pelvic region, suggesting intestinal mesentery involvement by a tumor with a neuroectodermal origin. The patient was pretreated with $20 \mathrm{mg}$ of phenoxybenzamine TDS and once stabilized, an exploratory laparotomy was performed through the left retroperitoneal approach. Vascular control was obtained at the infrarenal aorta and, then, at bilateral iliac arteries. The left ureter was explored and the tumor was cleared off it. Exploration of the renal artery and vein was done and revealed that the tumor was located far from them. A large highly vascular retroperitoneal mass fixed to the distal abdominal aorta was evident, which was cleared off the aorta, and the arterial branches were ligated. The tumor was completely resected without any detriment to the aorta and the need for vascular reconstruction. Exploration of the peritoneal cavity revealed no tumor involvement. The immunohistochemistry (IHC) staining of the specimen was positive for synaptophysin and chromogranin, and sustentacular cells showed positivity for S-100 protein (Figure 2). Histopathological examination confirmed the lesion to be a benign PGL measuring $11 \times 9.5 \times 9 \mathrm{~cm}$. There was also peripheral margin involvement, as well as capsular and perineural invasion without any lymphovascular invasion. Two examined dissected lymph nodes were tumor-free. The patient had an uneventful postoperative course. At the most recent follow-up, which was approximately 3 years after the surgery, she was found to be hypertensive. Laboratory data showed elevated plasma normetanephrine (770.1 pg/mL, normal range < $196 \mathrm{pg} / \mathrm{mL}$ ), plasma norepinephrine (1730 pg/mL, normal range up to $600 \mathrm{pg} / \mathrm{mL}), 24$-hour urine normetanephrine (1232 Ug/24h, normal range < $600 \mathrm{Ug} / 24 \mathrm{~h}$ ), and 24-hour urine norepinephrine (129 Ug/24h, normal range $<90$ $\mathrm{Ug} / 24 \mathrm{~h}$ ) levels. MIBG single-photon emission computed tomography (SPECT) scan revealed avid nodular uptake in both lungs, more prominent on the right side (Figure 3 ), compatible with tumor recurrence. The patient reported no respiratory complaints. The abdominal region was clear from the tumor on synchrotron radiation -CT (SR-CT) scan (Figure 4) and MIBG scan.

\section{Discussion}

PGLs are rare neuroendocrine tumors with high morbidity and mortality due to their multifocality and progres- 

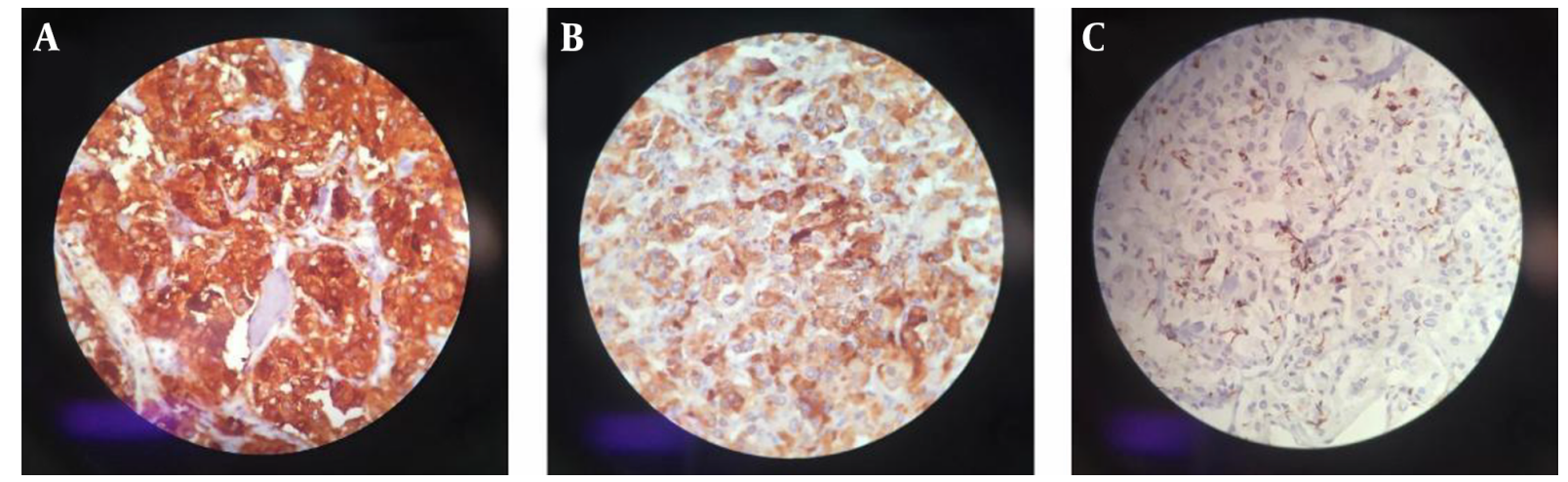

Figure 2. IHC staining with the original magnification of $40 \times$ showing positivity for A, synaptophysin; B, chromogranin; and C, S-100.
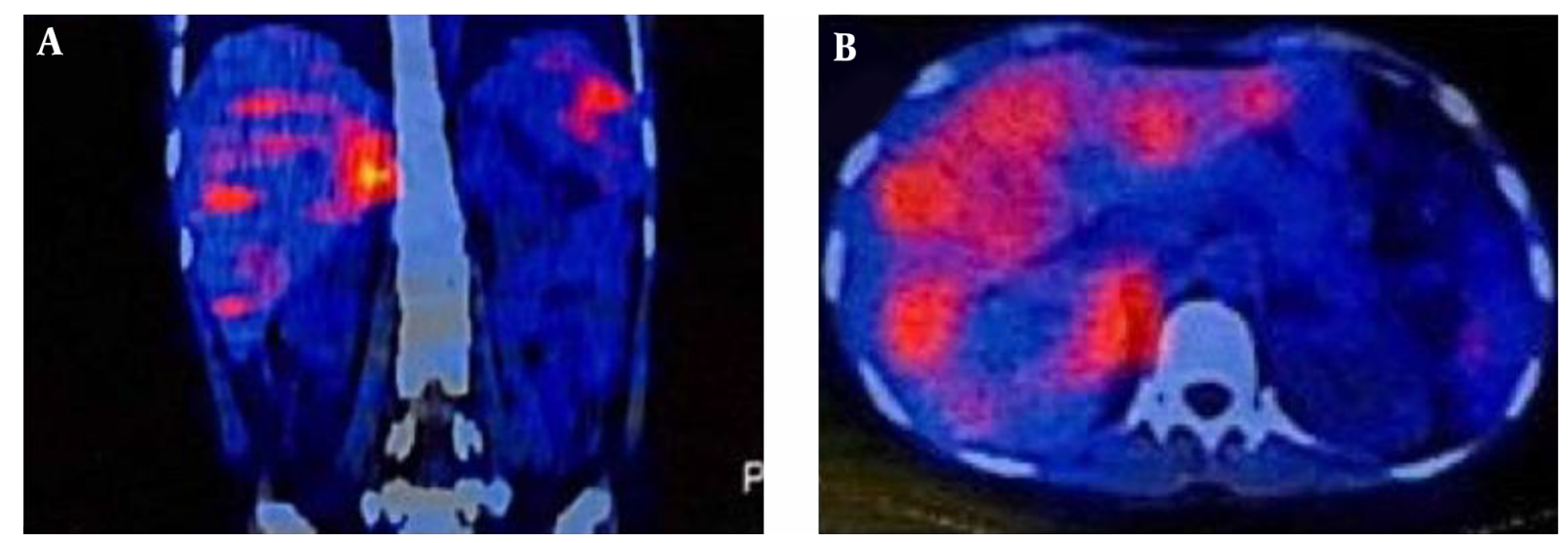

Figure 3. A, Coronal; and B, transverse sections of MIBG SPECT-CT scan showing avid nodular uptake in both lungs, more prominent on the right side.

sion (3). They carry a malignancy rate of about $10 \%$ and a 5-year survival rate of $<50 \%(4,5)$.

The classic triad of headache, sweating, and palpitations/tachycardia is uncommon, but when present, it increases the specificity to about 90\% (6). Catecholamine hypersecretion may be intermittent or continuous and is usually paroxysmal.

The diagnosis of a secretory PGL is usually made through measuring urinary and/or plasma fractionated metanephrines and catecholamines. It is worth noting that biochemical testing should be done in all PGL cases, even if clinically found to be nonfunctional. As other neuroendocrine tumors, elevated serum levels of antisera to neuron-specific enolase (NSE), chromogranin A, or vimentin can be useful to distinguish these tumors from the non-neuroendocrine ones (2).

Radiologic imaging also plays an important role in the evaluation of a PGL. Among available modalities, ultrasound, triple-phase helical CT, mag- netic resonance imaging (MRI) with gadoliniumdiethylenetriaminepentaacetic acid infusion (7), angiography, and radioisotope imaging using MIBG and 18F-fluoro-2-deoxyglucose positron emission tomography (FDG-PET) are commonly used. Histological examination reveals a thin capsule with polygonal or round epithelioid cells arranging in compact cell nests or trabecular patterns known as Zellballen (8). On IHC evaluation, chief cells usually show positivity for neuroendocrine markers such as synaptophysin, chromogranin, and NSE, and sustentacular cells are S-100 positive $(2,8)$.

Due to the catecholamine crisis leading to severe hypertension, an incisional or fine needle biopsy is contraindicated in a patient with suspected PGL unless there are negative biochemical testings or alpha-adrenergic blockers are prescribed. Furthermore, a biopsy can lead to severe hemorrhage or fibrosis with subsequent difficulty with definitive surgery $(1,9,10)$.

Locoregional PGLs can be managed by surgical re- 


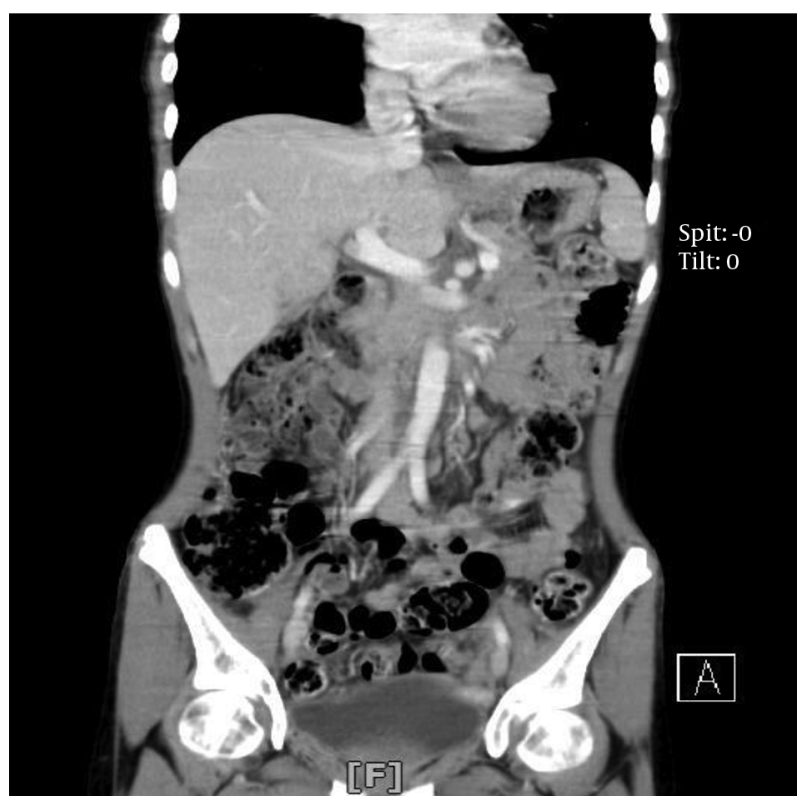

Figure 4. The abdominal region was clear from the tumor on the synchrotron radiation CT (SR-CT) scan.

section or radiation therapy. Generally, the preferred approach for localized PGLs below the neck and all catecholamine-secreting PGLs at any site is resection. Laparoscopic resection is often preferred, but with large $(>6$ $\mathrm{cm}$ ) tumors or tumors with a high risk of malignancy, exploratory laparotomy is suggested (11).

\subsection{Conclusions}

The diversity of manifestations and rarity of PGL can delay the diagnosis, which may lead to potential complications. This makes it compelling to include PGLs in differential diagnoses in a clinical setting compatible with catecholamine hypersecretion.

\section{Footnotes}

Authors' Contribution: Conception: Mohammad Mozaffar. Design and implementation: Adel Zeinalpour. Drafting of the manuscript: All authors. Collection and assembly of data: All authors. Final approval of manuscript: All authors. Accountable for all aspects of the work: All authors.
Conflict of Interests: The authors declare that there is no conflict of interest.

Funding/Support: This research received no specific grant from any funding agency in the public, commercial, or not-for-profit sectors.

Informed Consent: Written informed consent was obtained from the patient's parents (since she was a minor) and they agreed that the physicians could publish her disease-related article and any accompanying images with personal information were deleted.

\section{References}

1. Hu K, Persky MS. Multidisciplinary management of paragangliomas of the head and neck, Part 1. Oncology (Williston Park). 2003;17(983-93).

2. Gannan E, van Veenendaal P, Scarlett A, Ng M. Retroperitoneal nonfunctioning paraganglioma: A difficult tumour to diagnose and treat. Int J Surg Case Rep. 2015;17:133-5. doi: 10.1016/j.ijscr.2015.11.004. [PubMed: 26624503]. [PubMed Central: PMC4701798].

3. Asa SL, Ezzat S, Mete O. The diagnosis and clinical significance of paragangliomas in unusual locations. J Clin Med. 2018;7(9) doi: 10.3390/jcm7090280. [PubMed: 30217041]. [PubMed Central: PMC6162705].

4. Khare M, Gupta MK, Sharma UB, Garg KM. Paraganglioma with a very rare presentation. Indian J Pathol Microbiol. 2018;61(3):404-6. doi: 10.4103/IJPM.IJPM_334_17.[PubMed:30004066].

5. Higa B, Kapur U. Malignant paraganglioma of the pancreas. Pathology. 2012;44(1):53-5. doi: 10.1097/PAT.0b013e32834e42b6. [PubMed: 22157694].

6. Manger WM, Gifford RW. Clinical and experimental pheochromocytoma. 2nd ed. Cambridge: Blackwell Sciences; 1996.

7. Subramanian A, Maker VK. Organs of Zuckerkandl: Their surgical significance and a review of a century of literature. Am J Surg. 2006;192(2):224-34. doi: 10.1016/j.amjsurg.2006.02.018. [PubMed: 16860635].

8. Kliewer KE, Cochran AJ. A review of the histology, ultrastructure, immunohistology, and molecular biology of extra-adrenal paragangliomas. Arch Pathol Lab Med. 1989;113(11):1209-18. [PubMed: 2684087].

9. Vanderveen KA, Thompson SM, Callstrom MR, Young WJ, Grant CS, Farley DR, et al. Biopsy of pheochromocytomas and paragangliomas: potential for disaster. Surgery. 2009;146(6):1158-66. doi: 10.1016/j.surg.2009.09.013. [PubMed: 19958944].

10. Kubota K, Kato S, Mawatari H, Iida H, Akiyama T, Fujita K, et al. Risky endoscopic ultrasonography-guided fine-needle aspiration for asymptomatic retroperitoneal tumors. Dig Endosc. 2010;22(2):144-6. doi: 10.1111/j.1443-1661.2010.00939.x. [PubMed: 20447211].

11. Gill T, Adler K, Schrader A, Desai K, Wermers J, Beteselassie N. Extraadrenal pheochromocytoma at the organ of Zuckerkandl: A case report and literature review. Radiol Case Rep. 2017;12(2):343-7. doi: 10.1016/j.radcr.2016.12.009. [PubMed: 28491185]. [PubMed Central: PMC5417627]. 